\title{
Ion Temperature Measurements in SSPX
}

D.W. Auerbach, D.N. Hill, H.S. McLean

August 24, 2001

U.S. Department of Energy

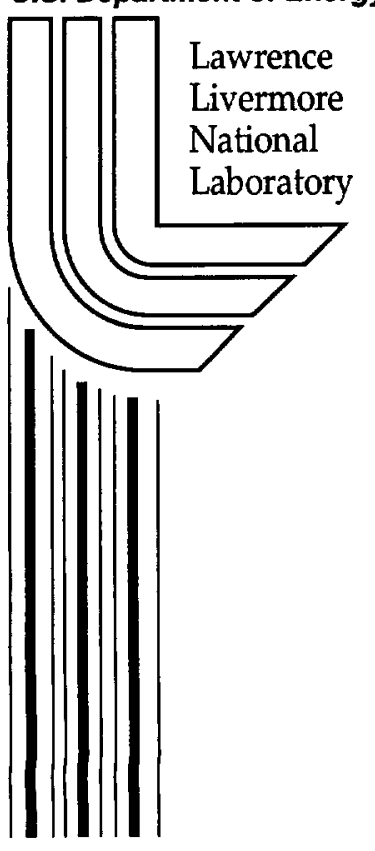




\section{DISCLAIMER}

This document was prepared as an account of work sponsored by an agency of the United States Government. Neither the United States Government nor the University of California nor any of their employees, makes any warranty, express or implied, or assumes any legal liability or responsibility for the accuracy, completeness, or usefulness of any information, apparatus, product, or process disclosed, or represents that its use would not infringe privately owned rights. Reference herein to any specific commercial product, process, or service by trade name, trademark, manufacturer, or otherwise, does not necessarily constitute or imply its endorsement, recommendation, or favoring by the United States Government or the University of California. The views and opinions of authors expressed herein do not necessarily state or reflect those of the United States Government or the University of California, and shall not be used for advertising or product endorsement purposes.

This is a preprint of a paper intended for publication in a journal or proceedings. Since changes may be made before publication, this preprint is made available with the understanding that it will not be cited or reproduced without the permission of the author.

This report has been reproduced directly from the best available copy.

Available electronically at http://www.doe.gov/bridge

Available for a processing fee to U.S. Department of Energy and its contractors in paper from

U.S. Department of Energy

Office of Scientific and Technical Information

P.O. Box 62

Oak Ridge, TN 37831-0062

Telephone: (865) 576-8401

Facsimile: (865) 576-5728

E-mail: reports@adonis.osti.gov

Available for the sale to the public from

U.S. Department of Commerce

National Technical Information Service

5285 Port Royal Road

Springfield, VA 22161

Telephone: (800) 553-6847

Facsimile: (703) 605-6900

E-mail: orders@ntis.fedworld.gov

Online ordering: http://www.ntis.gov/ordering.htm

OR

Lawrence Livermore National Laboratory

Technical Information Department's Digital Library

http://www.llnl.gov/tid/Library.html 


\title{
Ion Temperature Measurements in SSPX
}

\author{
D. W. Auerbach, D. N. Hill, and H. S. McLean \\ Lawrence Livermore National Laboratory
}

August 24, 2001

\begin{abstract}
The Ion Doppler Spectrometer instrument on the Sustained Spheromak Physics eXperiment is described, along with background about it's operation. Results are presented from recent experimental runs, and the data is compared to the results of simple statistical models of heat exchange in two species gasses.
\end{abstract}

\section{Introduction}

Many studies ([Fernández et al., 1990], [Gibson et al., 1995], [Hsu et al., 2000]) of ion temperatures have been performed on spheromaks, and all have seen very high ion temperatures during portions of the spheromaks lifetimes. These high temperatures are typically associated with magnetic reconnection events in the spheromak, such as when the flux lines of the plasma separate from those of the gun. An Ion Doppler Spectrometer (IDS) was built and installed at the Sustained Spheromak Physics eXperiment (SSPX) both to obtain ion temperature $\left(T_{i}\right)$ data for general performance assessment and to look at reconnection. This report describes background on how doppler spectroscopy works for temperature measurement, describes the details of the IDS instrument at SSPX, and then compares recent results to a simple statistical model of how two populations in a gas with distinct temperatures interact and exchange heat.

\section{Doppler Shift and Broadening of Spectral lines}

In a gas with weak molecular interactions, the particles (all of mass $m$ ) have a Maxwellian distribution of velocities. This is defined as

$$
f(v, T)=\left(\frac{m}{2 \pi k T}\right)^{\frac{3}{2}} e^{-\frac{m v^{2}}{2 k T}}
$$

dauerba1@sccs.swarthmore.edu

hilld@llnl.gov 
where $f(v, T)$ is the probability that a particle in a gas of temperature $T$ will have a velocity $v$ [Reif, 1965]. We are interested in using the Doppler shift of emitted radiation to measure this velocity distribution (and so measure temperature), so we want to integrate over the velocities perpendicular to the line of sight. This gives us

$$
f\left(v_{x}, T\right)=\left(\frac{m}{2 \pi k T}\right)^{\frac{1}{2}} e^{-\frac{m v_{x}^{2}}{2 k T}}
$$

where $x$ is the line of sight direction. This is a simple gaussian distribution.

If a particle emitting radiation of frequency $\nu$ is moving towards a detector at velocity $v$, the detected frequency $\nu_{d}$ is given by

$$
\nu_{d}=\nu\left(\frac{1+\frac{v}{c}}{1-\frac{y}{c}}\right)^{\frac{1}{2}}
$$

For small velocities $v$ equation 3 can be approximated as

$$
\nu_{d}=\nu\left(1-\frac{v}{c}\right)^{-1}
$$

from which we can find

$$
\frac{\Delta \nu}{\nu}=\frac{v}{c}
$$

Substituting $v$ in equation 5 into equation 2 gives us the distribution of frequencies

$$
f(\nu, T) \propto \exp \left[-\frac{m c^{2}\left(\frac{\Delta \nu}{\nu}\right)^{2}}{2 k T}\right]
$$

From equation 6, we can find that the half width half maximum of the dispersion (defined as half the spectral width of the gaussian at half the intensity of the maximum) is

$$
h w h m=\sqrt{\frac{(2 \ln 2) k T}{m c^{2}}} \lambda
$$

in units of wavelength. This dispersion is what is seen in a Doppler broadened spectral line.

\section{IDS-The Ion Doppler Spectrometer}

\subsection{Layout}

The Ion Doppler Spectrometer (IDS) for SSPX is based around a 1 meter focal length high resolution spectrometer, with a custom detector assembly attached to it. The spectrometer is an Instruments S.A. model THR1000 that has a 


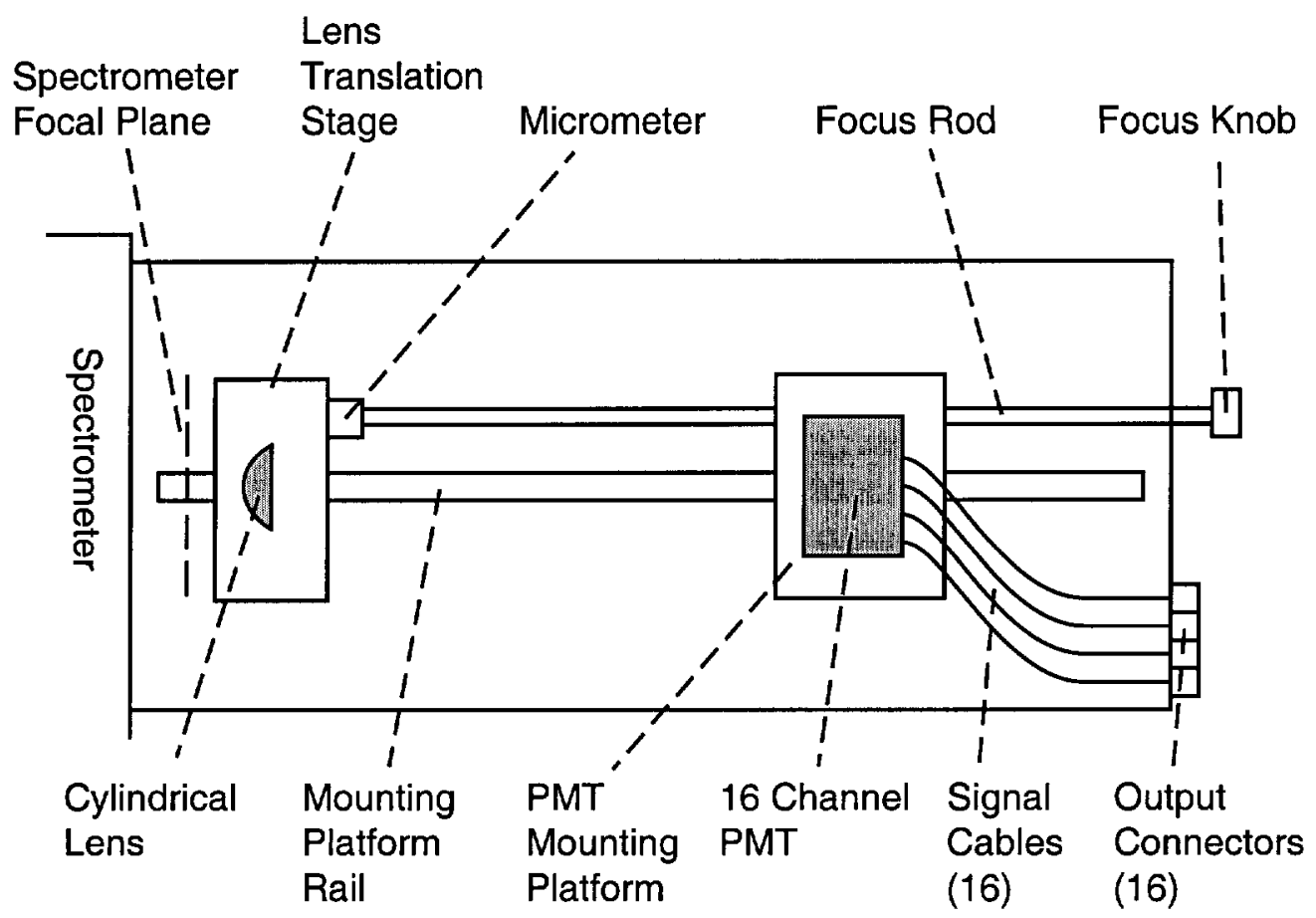

Figure 1: A detailed schematic of the IDS detector assembly

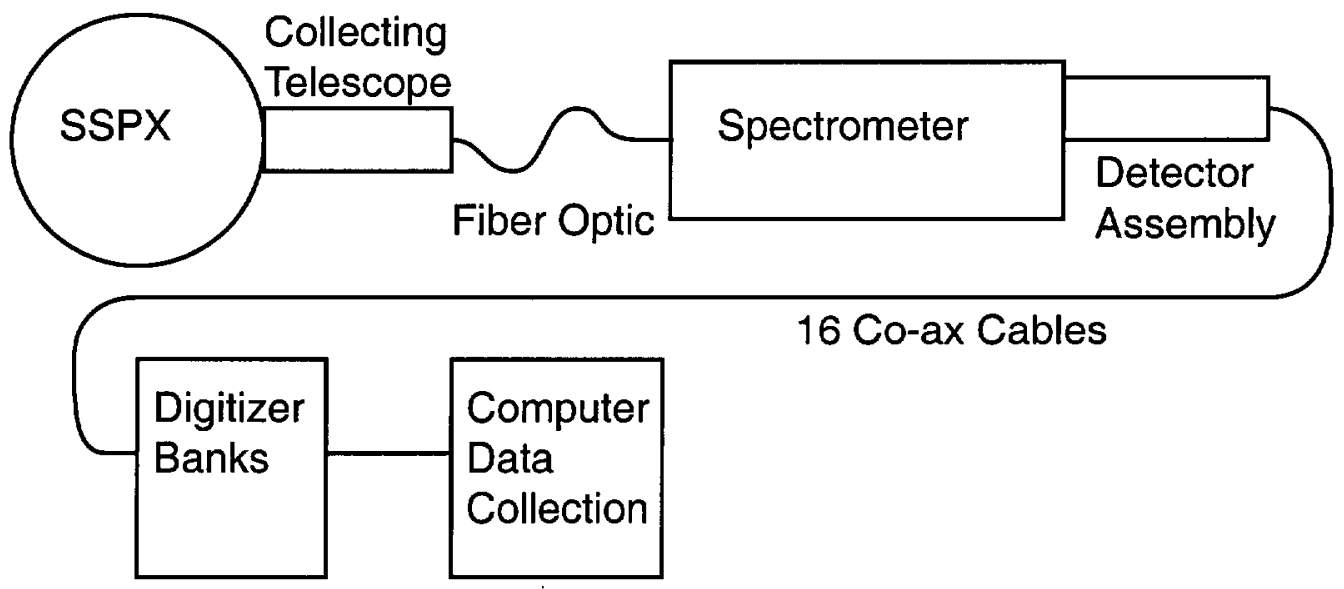

Figure 2: A schematic of the entire IDS system 


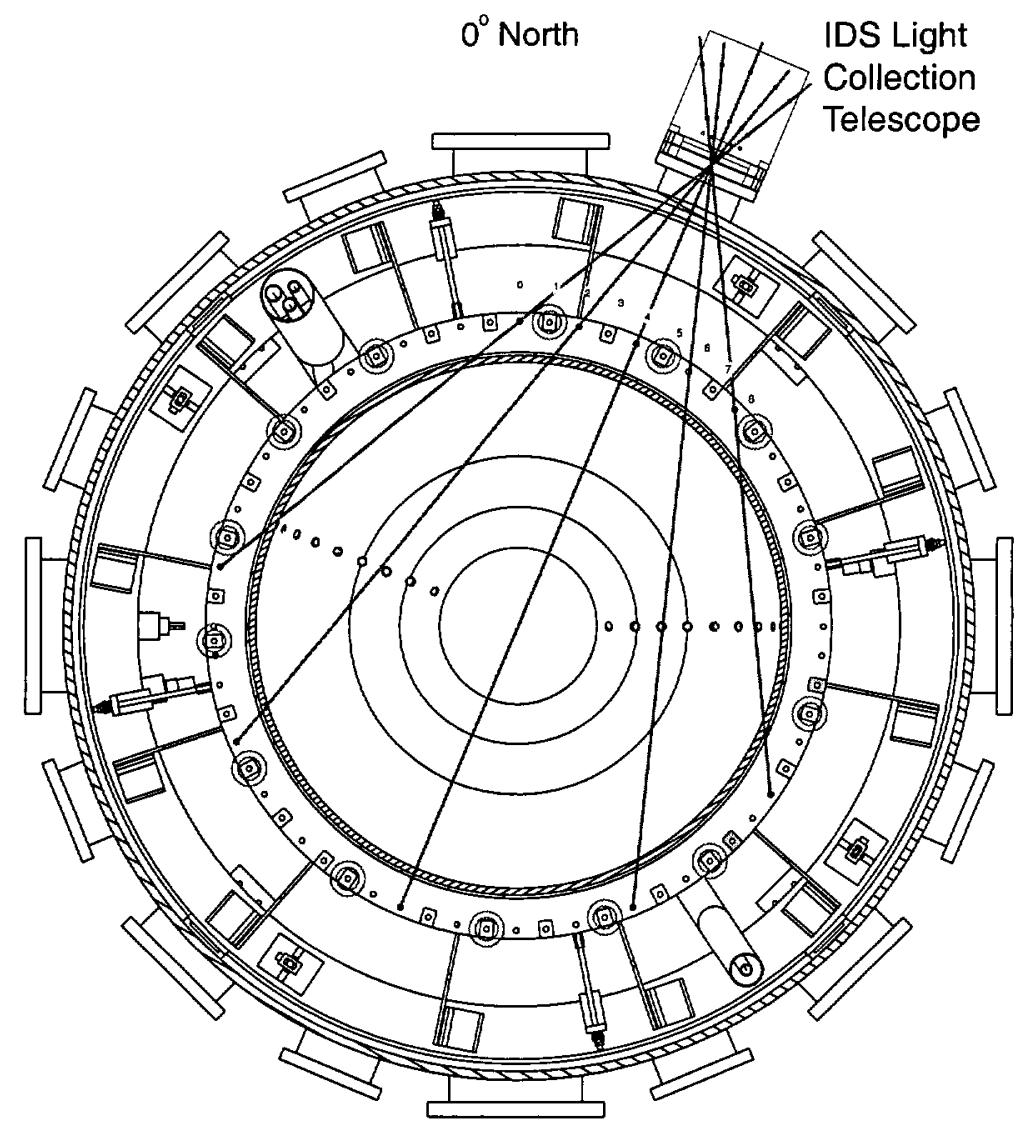

Figure 3: The 5 chords along which the IDS telescope can collect light. 
diffraction grating with a line density of 2400 lines/mm blazed for first order spectra between 300 and $600 \mathrm{~nm}$.

Light is collected for the IDS at a port on the vessel at the level of the diagnostics slot in the flux conserver. Mounted directly outside of the clear window of the port is a telescope with an 8 " focal length, 2" diameter collection lens, focused so that one end of a fiber optic bundle is exactly at the focal point of the lens. The result of this placement is that the fiber only collects light from a column of plasma the size of the lens. The orientation of the telescope is adjustable, so that the light can be collected along 5 different chords crossing the chamber (figure 3 ). This allows the temperature at different locations in the spheromak to be measured.

The fiber bundle used to carry the light from the collection telescope to the spectrometer is a custom built cable from Mitsubishi Cable Industries. It is composed of 24 individual $0.25 \mathrm{~mm}$ silica fibers in a circular arrangement (with a diameter of about $1.25 \mathrm{~mm}$ ) at the collection end and going to a linear arrangement at the output end, with the fibers in a single row of length $6 \mathrm{~mm}$. The linear end of the fiber is butted up against the input slit of the spectrometer and lined up carefully with the direction of the slit to maximize throughput.

The collected light then passes through the spectrometer and comes out at the detector box. The detector assembly is built around a Hamamatsu 16 channel photomultiplier tube (PMT), which consists of $160.8 \mathrm{~mm} \times 16 \mathrm{~mm}$ PMTs in a linear array. The tube is mounted in a light-tight box at the output of the spectrometer, with a cylindrical lens magnifying the spectrum and projecting it onto the PMT. Both the PMT and the lens are independently mounted on a rail assembly, so that they can be moved back and forth to adjust the magnification of the detector box. In addition, the lens platform is mounted on a micrometer controlled translation stage, with a shaft to the outside of the box allowing focus adjustment while the box is sealed and the PMT is powered. The final dispersion at the PMT, after magnification, is $0.28 \AA / \mathrm{mm}$.

The output from each of the 16 channels of the PMT is amplified by 16 separate amplifiers with a gain of $1 \mathrm{x}$ to $200 \mathrm{x}$, the level of which is controlled remotely by the SSPX operations and data acquisition computers. The coaxial signal lines are loaded with $10 k \Omega$ resistors as required by the PMT, and these result in an RC time constant for the signal path on the order of the sampling rate of the digitizers. The signal is then passed to 16 CAMAC digitizers, which record $8 \mathrm{~ms}$ of data at a $1 \mathrm{MHz}$ sampling rate beginning at $2 \mathrm{~ms}$ before the voltage is applied to the electrodes of the gun in the chamber. This data is then downloaded to a Unix system where analysis is performed.

\subsection{Alignment and Focusing}

Aligning the IDS was principally a matter of getting the proper strength signal to the digitizers with the least amount of noise. However, there were a large number of factors that needed to be addressed to ensure a high signal to noise ratio.

The first of these was to make sure that the light collection optic was properly 


\begin{tabular}{|l|l|}
\hline \multicolumn{2}{|c|}{ IDS Specs } \\
\hline Focal Length & 1 meter \\
\hline Grating Density & 2400 lines $/ \mathrm{mm}$ \\
\hline Dispersion & $0.28 \AA / \mathrm{mm}$ \\
\hline Channels & 16 \\
\hline Total Spectral View & $4.5 \AA$ \\
\hline Instrumental Profile Width & $0.55 \AA$ \\
\hline
\end{tabular}

Table 1: Important IDS Properties

focused onto the fiber optic. This meant making sure that the end of the fiber was at the focal point of the lens. This was checked by illuminating the opposite end of the fiber with a bright white light and looking at the size of the light spot projected by the lens. If the spot had the same diameter as the lens at a large distance from the lens, then the light was being properly collimated. Thus, the light collected by the fiber was only from the narrow column in front of the lens.

The second place where alignment was required was at the input slit. This was a fairly difficult task, because of the number of degrees of freedom in the system. The fiber is mounted at the slit on an X-Y translation stage, which allows movement of the end of the fiber across the slit and up and down the length of it. In addition, the angle of the linear end of the fiber is adjustable to get the fiber vertical. The method used to align this system was to set the spectrometer to a strong mercury line and illuminate the input end of the fiber with a mercury light. Care had to be taken so as not to expose the ends of the fiber to the mercury lamp for extended periods of time, as the strong UV component of the light produces ozone from the oxygen in the air which can darken the ends of the fiber and reduce fiber throughput. The detector was powered, and the voltage output of the center channel was monitored on a multimeter. With the slit opened to about $0.5 \mathrm{~mm}$, the angle of the fiber was adjusted to maximize the voltage of the center channel of the detector. The slit was then slowly narrowed to the desired width, and the fiber continuously adjusted using the translation stage to keep the output maximized. It was also necessary to adjust the wavelength setting of the spectrometer to keep the mercury line centered on the detector, as the adjustment of the slit size slightly affected the location of the line.

The output of the spectrometer also needed initial adjustment, and any changes in the magnification of the detector box would require this process again. A $15 \mathrm{x}$ magnification was selected by putting the detector $150 \mathrm{~mm}$ (11 +12 in the table) from the image plane of the spectrometer. The image plane was located approximately by projecting a spectral line on a white piece of paper and noting where the line was sharpest visually. The detector box was then sealed and the detector powered, and a spectral line was lined up with the detector. The focus knob was then adjusted to maximize the peak intensity of the spectral line at the detector, a process which also narrowed the spectral line as much as possible. 


\subsection{Operation}

Although the grating on the spectrometer is only blazed for 300 to $600 \mathrm{~nm}$, the response of the system goes down to around $230 \mathrm{~nm}$. Within this operational range of wavelengths, there are a number of emission lines that are typically bright in hot plasmas. Table 2 shows several emission lines, their wavelengths, and their widths at different temperatures.

Unfortunately for the purposes of ion temperature measurement, the vacuum chamber for SSPX is very clean, and impurity levels are quite low. This means that many of the predicted impurity lines are not actually visible. This initial design of the instrument called for a 1 inch collecting lens, and this had to be increased to a 2 inch lens to effectively illuminate the PMT. Even with this lens, the primary impurity line used, the OV line at $278.1 \mathrm{~nm}$, is only sometimes visible. The most reliable impurity line is the very bright OVI line, but as this is far into the UV at $120 \mathrm{~nm}$, a vacuum spectrometer would be needed to view this line.

\section{Data and Analysis}

Analysis of the collected data from the IDS is performed in the IDL program. The analysis assumes that both the line shape of the spectral line and the instrumental response function (the output of the instrument to a very narrow line) are both gaussian. For each data point (one time step) there are 16 channels of data across $4.5 \AA$. These data are fitted to a gaussian curve using a built in IDL routine. Since the dispersion of two convolved gaussian add in quadrature (see appendix B), the dispersion of the spectral line is calculated by

$$
\sigma_{\text {line }}=\sqrt{\sigma_{\text {out }}^{2}-\sigma_{\text {instrument }}^{2}}
$$

From equation 8 , the temperature can be calculated based on equations 6 and 7 in section 2. This procedure is repeated for each timestep, giving a time resolved ion temperature measurement of the shot.

The impurity lines are not alway visible during the entirety of the shot. An automatic preprocessing code in IDL picks out sections of good data and passes them to the analysis routine.

\subsection{Run Data}

Recent results from the IDS instrument, shown in figures 4 and 5, show initially very high ion temperatures, on the order of $1000 \mathrm{eV}$. These high temperatures cool very quickly, in times on the order of $0.1 \mathrm{~ms}$, to around $100 \mathrm{eV}$. For some shots, there are secondary rises in $T_{i}$ when the sustainment bank fires, which also cool down in times on the order of $0.1 \mathrm{~ms}$.

Figure 6 show a plot of an estimate of magnetic fluctuations in the toroidal 


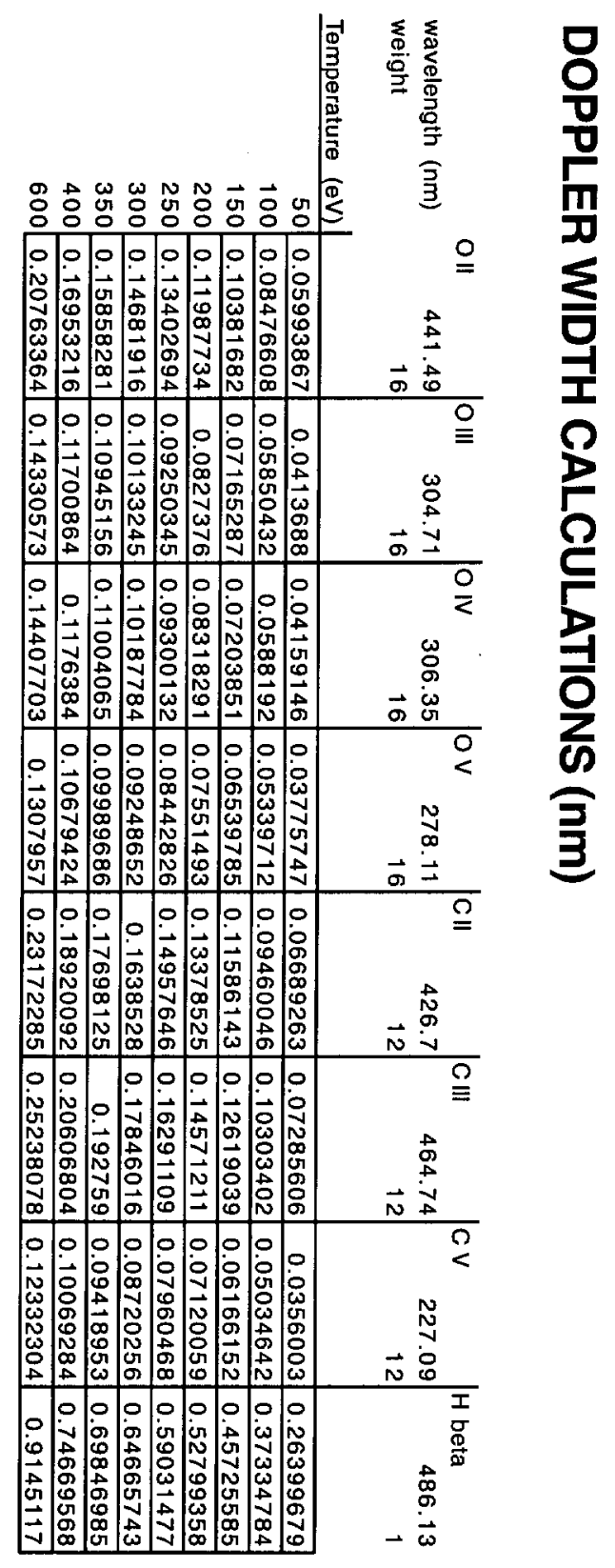

Table 2: The table of potential Doppler widths (FWHM, in $\mathrm{nm}$ ) for various atoms, emission lines, and ion temperatures. 


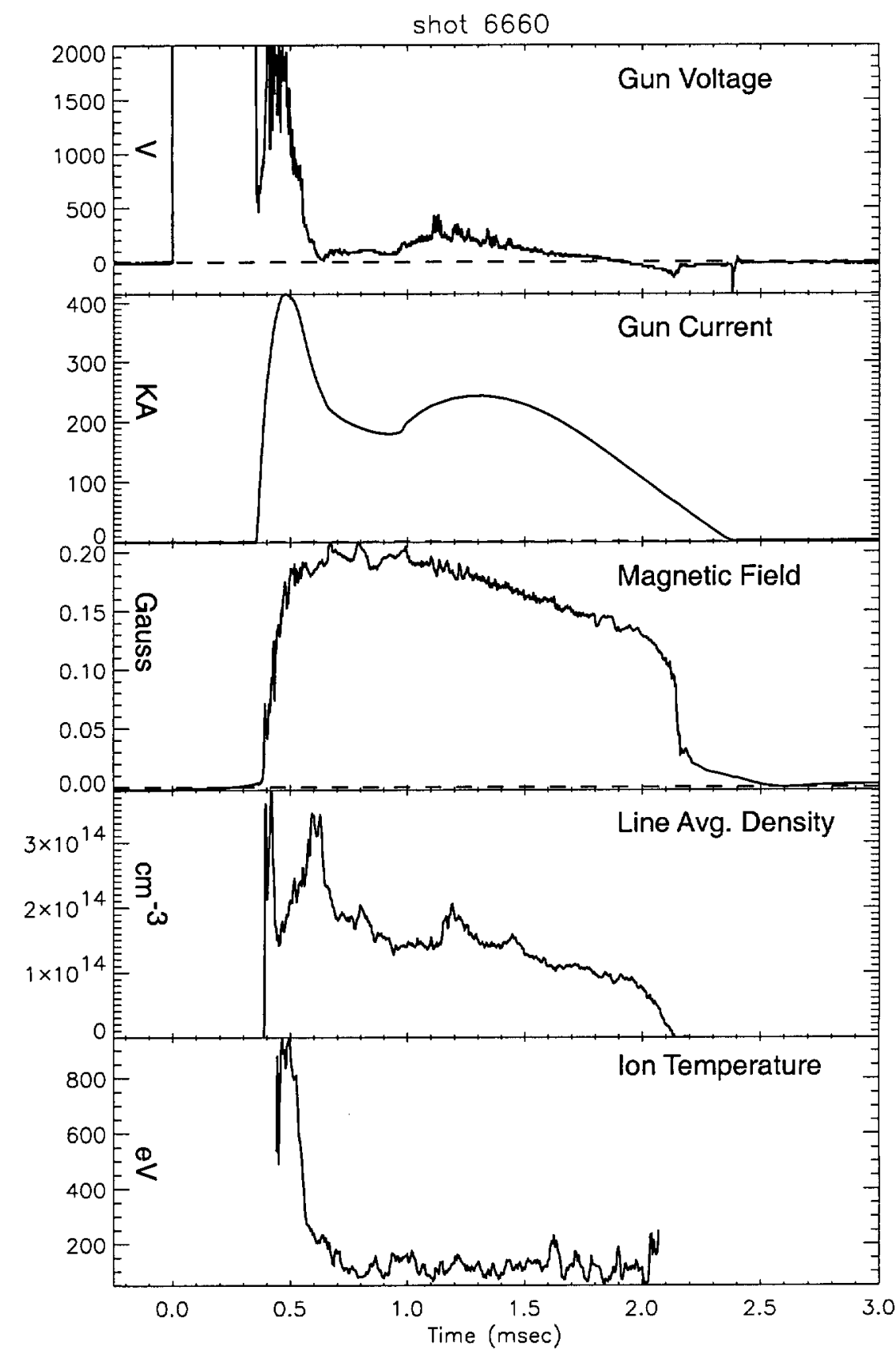

Figure 4: Example results from the IDS, with associated shot data 


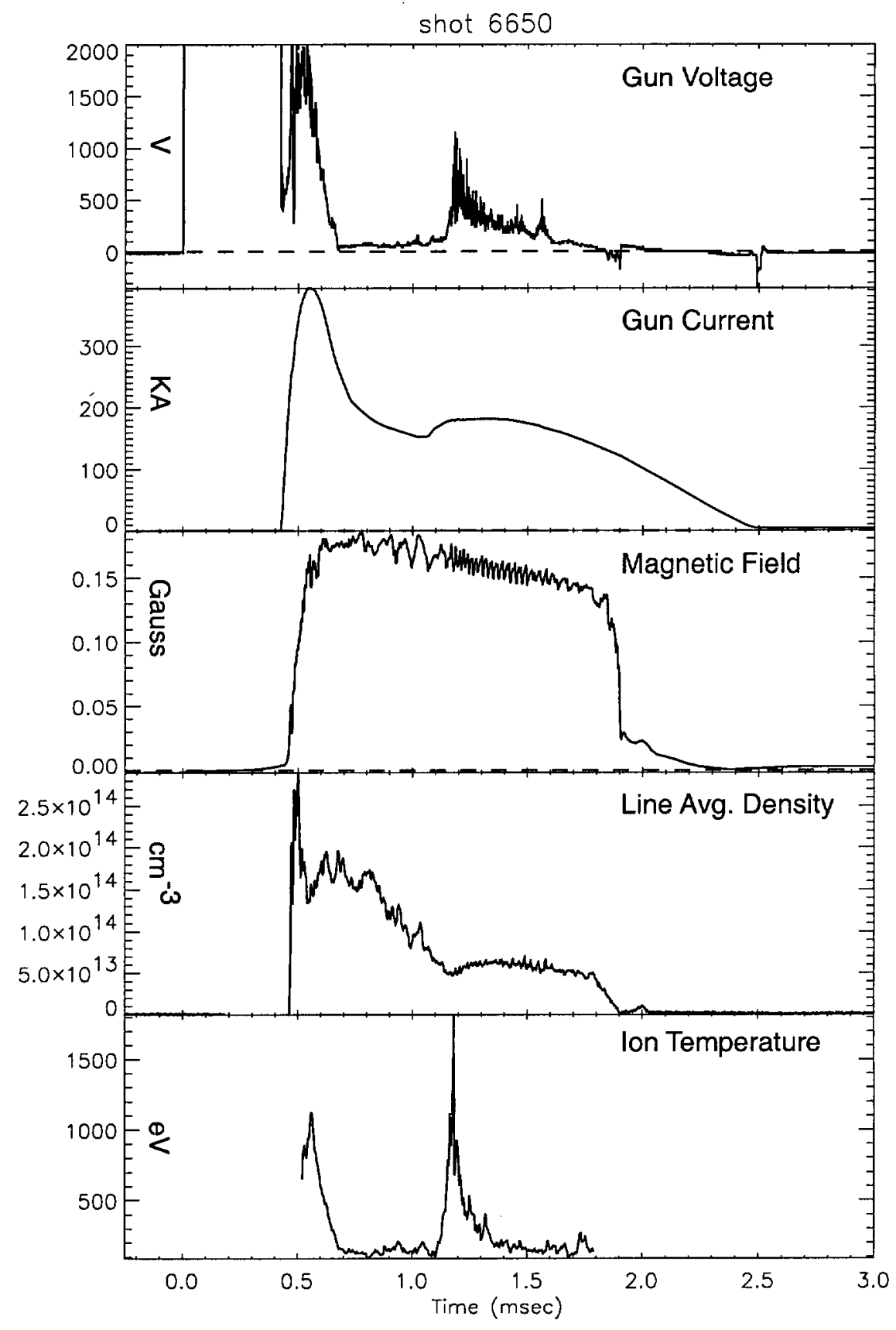

Figure 5: Example results from the IDS showing $T_{i}$ rise when the sustainment bank fires 


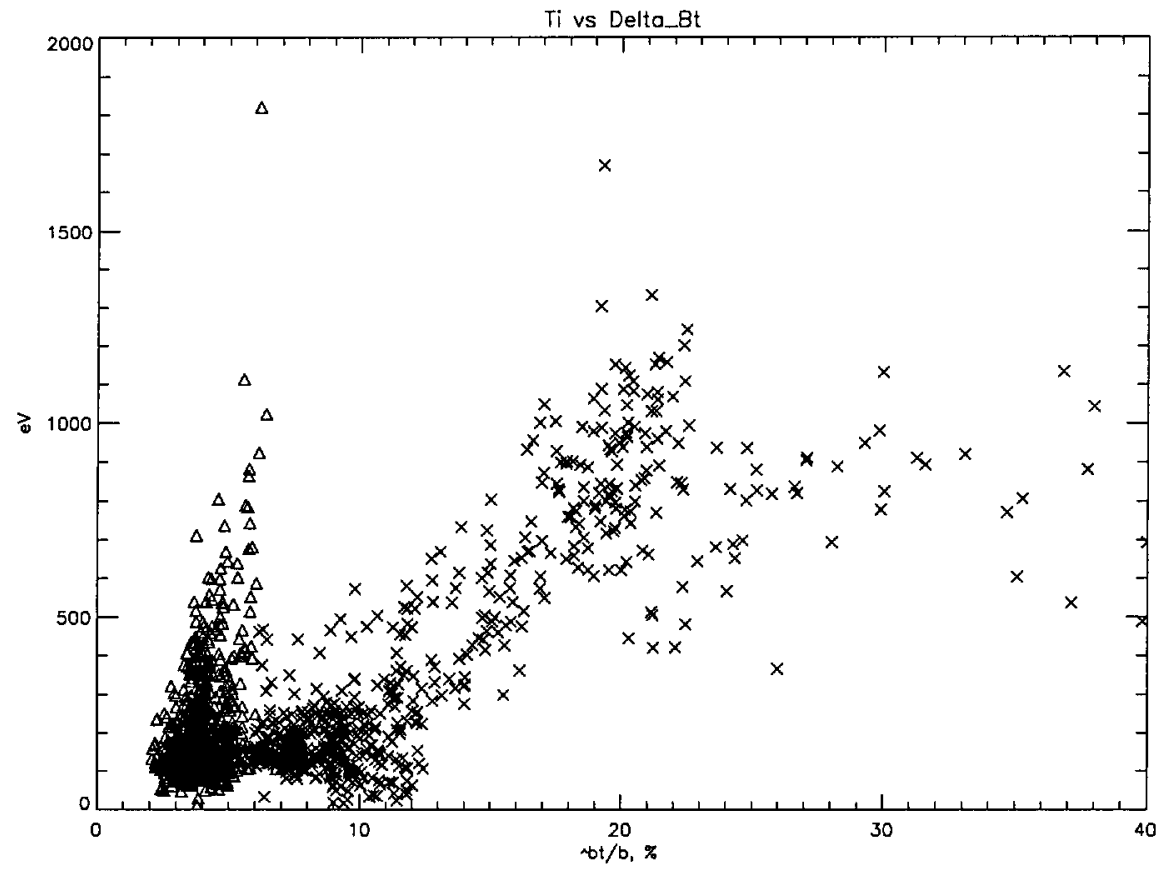

Figure 6: Plot of $\Delta B_{t} / B$ for eight recent shots. X's are times before $1 \mathrm{~ms}$, triangles are points after. 
field, $\Delta B / B$, versus $T_{i}$, for 8 different shots. $\Delta B / B$ is calculated by

$$
\frac{\Delta B}{B}=\frac{\sqrt{(B-\bar{B})^{2}}}{B}
$$

There is a correlation between high ion temperatures and magnetic fluctuations as expected, however there are some high temperatures that are not correlated with measured magnetic fluctuations. These particular measurements are only seen during the higher $T_{i}$ recorded after the firing of the sustainment bank. The measurements of magnetic field used to calculate $\Delta B / B$ are made at the magnetic axis of the plasma, and it is possible that other fluctuations may be responsible for the high temperatures, or that other mechanisms are operating.

\subsection{Models}

One basic model of cooling ions is that of two populations, ions and electrons, in equilibrium with themselves and with distinct temperatures. While the populations in SSPX are not in equilibrium because there is energy input into them, the time scale of temperature equilibration within the populations is much shorter than between them and thus the model should describe the experiment fairly well.

Spitzer [Spitzer, 1962] showed that the cooling rate for two distinct populations is given by

$$
\frac{d T}{d t}=\frac{T_{e}-t_{i}}{t_{e q}}
$$

where $t_{e q}$ is given by

$$
t_{e q}=\frac{3 m_{i} m_{e} k^{\frac{3}{2}}}{8(2 \pi)^{\frac{1}{2}} n\left(Z_{i}\right)^{2} \ln \Lambda}\left(\frac{T_{i}}{m_{i}}+\frac{T_{e}}{m_{e}}\right)^{\frac{3}{2}}
$$

Average SSPX shots have densities around $2 x 14^{14} \mathrm{~cm}^{-3}$, peak $T_{i}$ around 1000 $\mathrm{eV}, T_{e}$ around $100 \mathrm{eV}$. This predicts a time for $T_{i}$ to cool to $T_{e}$ of around 0.1 ms. Individual calculations for each shot predict cooling rates of 2 to 3 times faster than actually seen. This indicates that there is continued energy input into the ions even as they cool.

A similar technique can be used to look at energy dissipation rates. The energy in a monatomic gas is described by

$$
E=\frac{3}{2} n K T
$$

Since we can predict $\mathrm{dT} / \mathrm{dt}$ using equation 10 , we can find the energy dissipation associated with that cooling. Thomson scattering measurements on SSPX shots show $T_{e}$ around $100 \mathrm{eV}$, and since $T_{i}$ hovers around $200 \mathrm{eV}$, there must be continued energy input into the ions, and continuous energy dissipation through 
the electrons. Using the typical values described above, we find that typical power flow from the ions to the electrons and then out of the plasma is around $10 \mathrm{MJ} / \mathrm{s}$. A time resolved recording of $T_{e}$ would be more effective for this calculation, because a cooling rate could be calculated from the difference of $T_{e}$ and $T_{i}$ at all times, but as of yet $T_{e}$ is only measured via a single time point Thompson scattering system. 


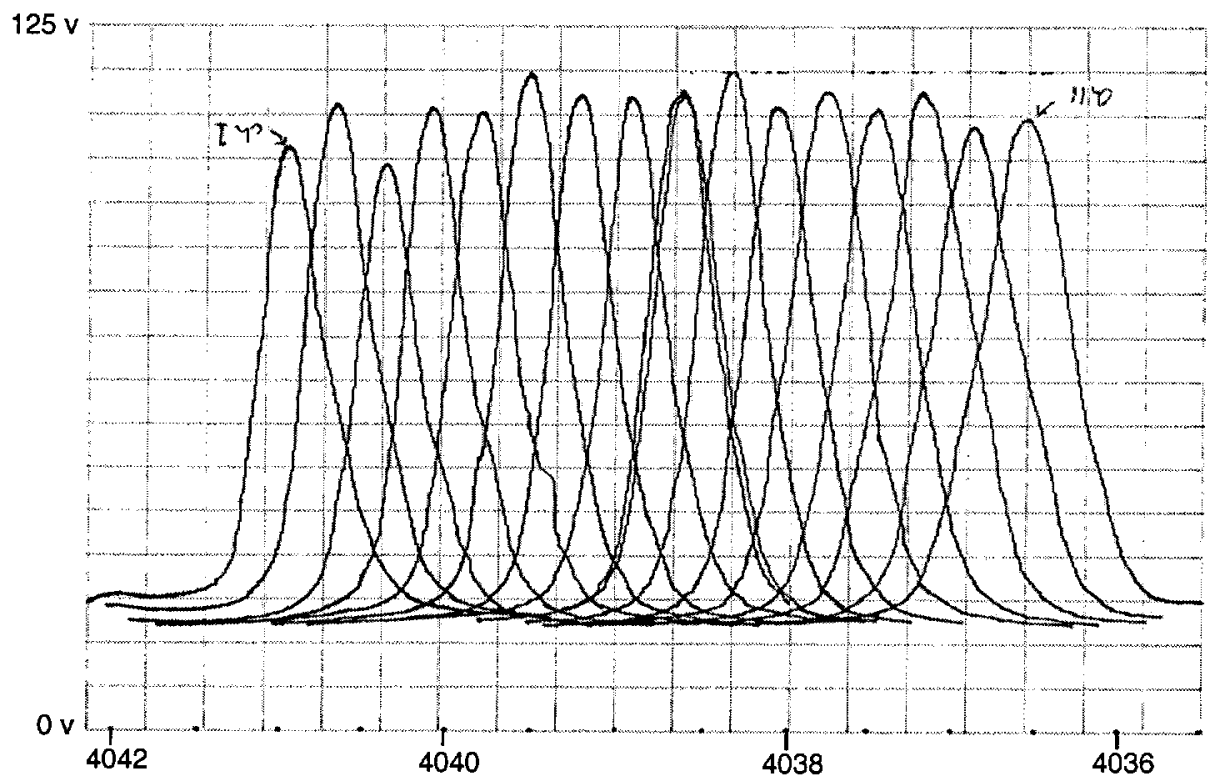

Figure 7: A plot of the response of each channel of the PMT detector to one specific mercury line at $4046 \AA$. The scale of the $\mathrm{y}$ axis is from 0 to $125 \mathrm{mV}$, and the $\mathrm{x}$ axis is in angstroms $(\AA)$. There is $0.3 \AA$ between channel peaks, and the FWHM of the lines is about $0.55 \AA$. The slit was set at $0.04 \mathrm{~mm}$.

\section{Appendix}

\section{A Spectrometer Calibration - Detector}

In order to use a multi channel detector such as the 16 channel PMT in the IDS detector box, it was necessary to calibrate and normalize each channel's intensity of response to the same signal. To do this, the same mercury line from a calibration lamp was passed over each of the detectors one by one, with the signal output hooked up to an analog plotter. The peak height of each channel was then measured, and a calibration factor was generated for each channel to normalize the output of the detectors. This factor was used as input to the data analysis routine, so that no direct manipulation of the data was required outside of that routine.

\section{B Convolution of Gaussian}

Because the IDS instrument has an instrumental profile, the spectrum at the output is different from the input spectrum. The actual output is a convolution of the input spectra and the instrument profile. To see this, we consider in figure 7 the detected response of the IDS instrument to a very narrow line (a 


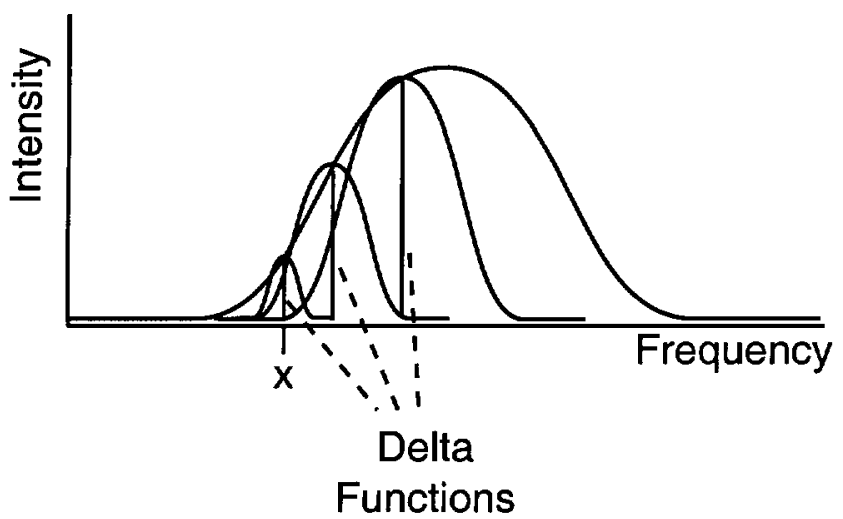

Figure 8: A broad spectral line broken down into delta functions, with the IDS instrumental response to each individual delta function superimposed

cold mercury line). We can think of this as the response of the instrument to a delta function, since the width of the mercury line is much smaller than the measured line width of $0.55 \AA$. If we then consider the input signal as being a sum of very close together delta functions, we can see that the total system response to the input signal will be the sum of responses to each of the delta functions (see figure 8). If the intensity of the delta function at a specific wavelength $\lambda$ is given by $\operatorname{Int}(\lambda)$, and the instrumental response is given by $R(d)$, where $d$ is the displacement from the center of the symmetric response function, then the intensity at a specific $\lambda=x$ at the output can be represented as the sum

$$
I(x)=\cdots+\operatorname{Int}(x) \cdot R(0)+\operatorname{Int}(x+\delta) \cdot R(\delta)+\operatorname{Int}(x+2 \delta) \cdot R(2 \delta)+\cdots
$$

where $\delta$ is the infinitesimal displacement to the next delta function. In the limit as $\delta$ becomes very small, is is clear that

$$
I(x)=\int_{-\infty}^{\infty} \operatorname{Int}(x+\delta) \cdot R(-\delta) d \delta
$$

This is a standard convolution integral.

If both the input spectrum and the instrumental response are gaussian (or can be approximated as gaussian), this convolution integral which represents intensity at the output of the spectrometer has nice properties that can be exploited to make the data analysis for finding ion temperatures easier. We.will assume that the intensity of the incoming spectrum is given by

$$
\operatorname{Int}(\lambda)=C \cdot \exp \left[-\frac{1}{2}\left(\frac{\lambda-\lambda_{0}}{\sigma_{i}}\right)^{2}\right]
$$


and the instrumental response has the form

$$
R(\delta)=C \cdot \exp \left[-\frac{1}{2}\left(\frac{\delta}{\sigma_{r}}\right)^{2}\right]
$$

We will ignore constants in the following derivation and include them all in $C$, a constant whose definition will change throughout the calculation.

From equation 14, with a change of variable $\lambda=\lambda-\lambda_{0}$, we can see that the output intensity is given by

$$
\begin{aligned}
I(\lambda) & =C \int_{-\infty}^{\infty} \exp \left[-\frac{1}{2}\left(\frac{\lambda+\delta}{\sigma_{i}}\right)^{2}\right] \exp \left[-\frac{1}{2}\left(\frac{-\delta}{\sigma_{r}}\right)^{2}\right] \\
& =C \int_{-\infty}^{\infty} \exp \left[-\frac{1}{2}\left(\frac{\delta^{2}\left({\sigma_{r}}^{2}+{\sigma_{i}}^{2}\right)+2 \lambda \delta{\sigma_{r}}^{2}+\lambda^{2}{\sigma_{r}}^{2}}{\sigma_{r}{ }^{2} \sigma_{i}{ }^{2}}\right)\right] d \delta
\end{aligned}
$$

At this point, we complete the square in the exponent, giving

$$
I(\lambda)=C \int_{-\infty}^{\infty} \exp \left[-\frac{1}{2}\left(\frac{\left(\frac{{\sigma_{r}}^{2}+\sigma_{i}{ }^{2}}{\sigma_{r} \sigma_{i}} \delta+\frac{\lambda \sigma_{r}}{\sigma_{i}}\right)^{2}+\lambda^{2}}{\sigma_{r}^{2}+\sigma_{i}^{2}}\right)\right] d \delta
$$

Making the substitution

$$
u=\frac{{\sigma_{r}^{2}}^{2}+\sigma_{i}^{2}}{\sigma_{r} \sigma_{i}} \delta+\frac{\lambda \sigma_{r}}{\sigma_{i}}, \quad d u=\frac{\sigma_{r}^{2}+\sigma_{i}^{2}}{\sigma_{r} \sigma_{i}}
$$

and again dropping constants, gives

$$
I(\lambda)=C \int_{-\infty}^{\infty} \exp \left[-\frac{1}{2}\left(\frac{u^{2}+\lambda^{2}}{{\sigma_{r}}^{2}+\sigma_{i}^{2}}\right)\right] d u
$$

Finally, since the integral of a gaussian over all space is a constant, we can pull out the $\lambda$ dependence from the integral, leaving just a constant behind. Replacing $\lambda$ with $\lambda-\lambda_{0}$ as our original variable, we have an instrumental output intensity of

$$
I(\lambda)=C \cdot \exp \left[-\frac{1}{2}\left(\frac{\left(\lambda-\lambda_{0}\right)^{2}}{\sigma_{r}^{2}+\sigma_{i}{ }^{2}}\right)\right]
$$

Thus, we see that when two gaussian are convolved, their $\sigma$ 's add in quadrature. This enormously simplifies deconvolution. If the output spectrum from a spectrometer is a gaussian, and the instrumental function is also a gaussian, than the $\sigma$ of the incoming gaussian line profile is simply

$$
\sigma_{\text {in }}=\sqrt{{\sigma_{\text {out }}^{2}}^{2}-\sigma_{r}^{2}}
$$




\section{References}

[Fernández et al., 1990] Fernández, J. C., Jarboe, T. R., Knox, S. O., Henins, I., and Marklin, G. J. (1990). Ion heating and current drive from relaxation in decaying spheromaks in mesh flux conservers. Nuclear Fusion, 30:67.

[Gibson et al., 1995] Gibson, K. J., Gee, S. J., and Rusbridge, M. G. (1995). Ion energy measurements in the sphex spheromak. Plasma Physics and Controlled Fusion, 37(1):31.

[Hartog and Holly, 1997] Hartog, D. J. D. and Holly, D. J. (1997). A simple, low-cost, versatile charge-coupled device spectrometer for plasma spectroscopy. Review of Scientific Instruments, 68(1):1036.

[Hill et al., 2000a] Hill, D. N. et al. (2000a). Overview of spheromak experiments on sspx. APS 2000 Poster Session.

[Hill et al., 2000b] Hill, D. N. et al. (2000b). Spheromak formation studies in sspx. In 18th International Atomic Energy Agency Fusion Energy Conference. IAEA.

[Hsu et al., 2000] Hsu, S. C., Fiksel, G., Carter, T. A., Ji, H., Kulsrud, R. M., and Yamada, M. (2000). Local measurement of nonclassical ion heating durring magnetic reconnection. Physical Review Letters, 84(17):3859.

[Mayo et al., 1993] Mayo, R. M., Hurlburt, D. J., and Fernández, J. C. (1993). Ion temperature profile deconvolution and corrections to confinement parameters in spheromaks. Physics of Fluids B, 5(11):4002.

[McLean et al., 2001] McLean, H. S. et al. (2001). Plasma diagnostics for the sustained spheromak physics experiment. Review of Scientific Instruments, $72(1): 556$.

[Ono et al., 1996] Ono, Y., Yamada, M., Akao, T., Tajima, T., and Matsumoto, R. (1996). Ion acceleration and direct ion heating in three-componant magnetic reconnection. Physical Review Letters, 76:3328.

[Reif, 1965] Reif, F. (1965). Fundamental os Statistical and Thermal Physics. McGraw-Hill, New York.

[Spitzer, 1962] Spitzer, L. (1962). Physics of Fully Ionized Gasses 2nd Edition. Interscience, New York.

This work was performed under the auspices of the U.S. Department of Energy by the University of California, Lawrence Livermore National Laboratory under Contract No. W-7405-Eng-48. 